\title{
An External CAM Therapy (Tian Jiu) versus Placebo in Treatment of Allergic Rhinitis: A Pilot Single-Blinded, Three-Arm, Randomized Controlled Study
}

\author{
Liang Dai, Linda L. D. Zhong $\mathbb{D}$, Wai Kun, Wai Ching Lam $(\mathbb{D}$, Zhen Yang, Tao Huang, \\ Huaixue Mu iD, and Zhao-xiang Bian iD \\ Hong Kong Chinese Medicine Clinical Study Centre, School of Chinese Medicine, Hong Kong Baptist University, Hong Kong, China \\ Correspondence should be addressed to Zhao-xiang Bian; bianzxiang@gmail.com
}

Received 6 August 2018; Accepted 10 March 2019; Published 14 April 2019

Academic Editor: Gerhard Litscher

Copyright (c) 2019 Liang Dai et al. This is an open access article distributed under the Creative Commons Attribution License, which permits unrestricted use, distribution, and reproduction in any medium, provided the original work is properly cited.

\begin{abstract}
Allergic rhinitis (AR) is one of the common allergic diseases in clinical practice and significantly impairs the quality of life (QoL) of patients. The conventional treatments are not satisfactory because of various reasons. Tian Jiu (TJ) therapy is a characteristic external intervention of complementary and alternative medicine (CAM) and has been widely utilized in the management of AR. However, the evidences resulted from current studies were generally poor due to high risk of bias. Therefore, we conducted this rigorous designed, single-blinded, three-arm, randomized controlled study to evaluate the efficacy and safety of TJ therapy on AR. Totally 138 AR patients were enrolled. The TJ group and placebo group received 4-week treatment with either TJ or placebo patches for 2 hours each time applied to Dazhui (GV 14), bilateral Feishu (UB 13), and bilateral Shenshu (UB 23) one session per week and then underwent a 4-week follow-up. The waitlist group received no treatment during the corresponding treatment period, but would be given compensatory TJ treatment in the next 4 weeks. The primary outcome was the change of the Total Nasal Symptom Score (TNSS) after treatment. The secondary outcomes included the changes of Rhinoconjunctivitis Quality of Life Questionnaire (RQLQ) and rescue medication score (RMS). After treatment, the total TNSS in TJ group was significantly reduced compared with baseline, but showed no statistical meaning compared with placebo. Among the four domains of TNSS, the change of nasal obstruction exhibited statistical difference compared with placebo group. The total RQLQ score in TJ group was significantly reduced compared with both placebo and waitlist groups. The needs of rescue medications were no distinct difference between two groups. In summary, this study showed potential effectiveness of TJ therapy in improving nasal obstruction symptoms and QoL of AR patients.
\end{abstract}

\section{Introduction}

Allergic rhinitis (AR), affecting $10 \%$ to $20 \%$ of the population globally, is one of the common allergic diseases in clinical practice [1]. In China, based on the epidemiological surveys conducted in major cities, the prevalence of selfreported AR had increased from $11.1 \%$ to $17.6 \%$ in a 6-year period (2005 to 2011) [2, 3]. Although AR is not a lifethreatening disease, the classical nasal and ocular symptoms, including nasal obstruction and pruritus, eyelid edema, and asthenia, significantly impair the quality of life (QoL) of patients [4-7]. Routine symptomatic pharmacotherapies contain $\mathrm{H}_{1}$-antihistamines, intranasal glucocorticoids, and leukotriene-receptor antagonists [8], while it has been reported that only one-third of adults experienced satisfactory relief after treatment [9]. For the non-responders, immunotherapy would be considered in next step. However, the optimal use, appropriate initiation timing, and proper duration of immunotherapy are still difficult to determine [8] Therefore, many patients would seek help from complementary and alternative medicine (CAM), especially Traditional Chinese Medicine (TCM) in East Asia [10-12].

Tian Jiu (TJ), also known as "Chinese Medicine moxibustion" or "acupoint Chinese Medicine patching", is a typical external intervention of TCM and has been utilized in multiple respiratory diseases in China for a long history [13]. 
During TJ treatment, pre-prescribed acupoints were attached by Chinese Medicine patches. On the foundation of TCM theory, this intervention could regulate the functions of meridians and viscera and thereby invigorate physical institution so as to prevent and treat diseases [14]. In addition, the abnormal body institution is considered to be the internal reason of AR attack according to TCM concept; thus it is reasonable to apply TJ in management of AR [15]. The specific mechanisms of the effect of TJ therapy are still not clean, but multiple systems were reported to be involved [16]. Animal researches reported that the $\mathrm{TJ}$ could reduce the level of peripheral blood eosinophils and serum immunoglobulin E ( $\operatorname{IgE}$ ), inhibit mast cell degranulation, decrease the production of inflammatory cytokines, and suppress lymphocyte immune response [1720]. Previous meta-analysis has reported the efficacy and safety of AR in TJ treatment. TJ alone or in combination with Western medicine showed better effectiveness than placebo or Western medicine, respectively. However, the conclusion resulted from trials with high risk bias and should be interpreted with caution [21].

Therefore, for the purpose of further evaluating the effectiveness of TJ therapy in the management of AR, we conducted this rigorous designed trial by evaluating the generally accepted symptoms scales, Total Nasal Symptom Score (TNSS), and Rhinoconjunctivitis Quality of Life Questionnaire (RQLQ). The hypothesis was that TJ group would significantly alleviate symptoms and improve QoL of AR patients when compared with placebo and waitlist groups.

\section{Materials and Methods}

2.1. Study Design. It was a prospective, randomized, singleblinded, controlled clinical study. A total of 138 participants were included from the public by advertisements on website and local newspaper. After a one-week run-in period, qualified participants were randomly allocated to TJ arm, placebo arm, and waitlist arm. TJ group and placebo group received either Chinese Medicine patches or placebo patches one session per week continuously for 4 weeks, respectively. After treatment period, another 4-week follow-up was set to evaluate the sustained effects. The waitlist group was given no intervention during the first 4 weeks, but, after the waiting period, participants in waitlist group were given compensatory TJ treatment for 4 weeks. Based on this design, the effect of TJ and placebo could be evaluated simultaneously. All groups were evaluated identically during the whole study. The total study period was nine weeks.

The study protocol was approved by the Hong Kong Baptist University Ethics Committee on the Use of Human Subjects for Teaching and Research (Approval no. HASC/1314/0241). The protocol has been registered with the identifier NCT02470845 (17 May 2015) at ClinicalTrials.gov and published in Trials [22]. Every included patient signed the Informed Consent voluntarily. The checklists for items in CONSORT 2010 and STRICTOM 2010 were given in Supplement 1 and Supplement 2, respectively [23, 24]. The research was supported by the Marcoda Co. Ltd., which had no role in the protocol design, study implementation, data collection, and manuscript preparation.

\subsection{Participants}

Setting. The study was conducted in the research and clinical centers, School of Chinese Medicine, Hong Kong Baptist University (HKBU).

Inclusion Criteria. Patients who met all following criteria were defined as qualified participants: (1) both genders aged at least 18 years old; (2) having intermittent or persistent AR; (3) severity beyond mild (TNSS $\geq 8$ ). The diagnostic standard for AR was based on ARIA criteria: typical history of allergic symptoms plus diagnostic tests [20]. The typical symptoms include rhinorrhoea, sneezing, nasal obstruction, and pruritus. Diagnostic tests, such as skin prick test, radio allergo sorbent test (RAST), and nasal provocation, need to demonstrate allergen-specific IgE in the skin or the blood.

Exclusion Criteria. Patients who met any of the following criteria were excluded: (1) concomitant with allergic asthma and moderate to severe atopic dermatitis; (2) concomitant with any autoimmune diseases; (3) concomitant with severe chronic inflammatory diseases; (4) history of anaphylactic reactions; (5) hypersensitivity to cetirizine or related drugs; (6) application of specific immunotherapy during the past 3 years or planned in the next 2 years; (7) pregnancy or lactation.

\subsection{Interventions}

Medications. All included participants were informed to stop taking related medications, and the application of emergency medications was only allowed when the AR symptoms were uncontrollable. Patients in the TJ group and placebo group were treated with either Chinese Medicine patches or placebo patches on five identical acupoints on the back, respectively. The patches were bought from licensed medical device supplier. The ingredients of the Chinese Medicine patch contained Bai Jie Zi (Sinapis semen), Yan Hu Suo (Corydulis rhizoma), Zhi Gan Sui (Kansui radix), Xi Xin (Asari radix et rhizama), and synthesized Artificial She Xiang (Moschus artifactus). The composition was modified on the foundation of previous systematic review, preliminary survey among experienced TCM practitioners, and routine TJ formula used in HKBU clinics for more than ten years $[21,25,26]$. The composition and the action of each herb in the TJ Chinese Medicine patches were listed in Table 1.

In order to make high quality Chinese Medicine patch, the contents of crude medicinal materials were determined by high-performance liquid chromatography (HPLC). This method was well validated in terms of linearity, precision, accuracy, recovery, and stability. All calibration curves had good linearity and were shown in Supplement 3. The precision, accuracy, recovery, and stability were satisfactory. The established HPLC method was successfully applied to the simultaneous determination of euphadienol, asarinin, sinapine thiocyanate, and tetrahydropalmatine. Representative chromatograms of the standard solution and samples were shown in Supplement 4. All the contents were summarized in Supplement 5. 
TABLE 1: Composition and action of Tian Jiu treatment formula.

\begin{tabular}{|c|c|c|}
\hline Ingredients & $\%$ & Action \\
\hline \multirow[t]{2}{*}{ Sinapis Semen } & \multirow[t]{2}{*}{8.79} & $\begin{array}{l}\text { TCM: (1) To warm the lung and sweep phlegm, (2) To } \\
\text { disinhibit qi, (3) To dissipate binds and unblock } \\
\text { collaterals to relieve pain }\end{array}$ \\
\hline & & $\begin{array}{l}\text { Pharmaceutical study: (1) Antibacterial effect, (2) Skin } \\
\text { stimulating effect, (3) Effect on gastric secretion, (4) } \\
\text { Emetic effect, (5) Regulation of blood pressure, (6) } \\
\text { Anti-lipid peroxidation, (7) Expectorant effect }\end{array}$ \\
\hline \multirow[t]{2}{*}{ Corydulis Rhizoma } & \multirow[t]{2}{*}{11.01} & $\begin{array}{l}\text { TCM: (1) To activate blood, (2) To move qi, (3) To } \\
\text { relieve pain }\end{array}$ \\
\hline & & $\begin{array}{l}\text { Pharmaceutical study: (1) Effect on the CNS (analgesic } \\
\text { effect, hypnotic effect, sedative and tranquilizing } \\
\text { effects), (2) Muscular relaxant effect, (3) Cardiovascular } \\
\text { effect, (4) Effect on gastric secretion and on urination, } \\
\text { (5) Effect on pituitary-adrenocortical function }\end{array}$ \\
\hline \multirow[t]{2}{*}{ Kansui Radix } & \multirow[t]{2}{*}{6.56} & $\begin{array}{l}\text { TCM: (1) To expel retained fluid by purgation, ( } 2 \text { ) To } \\
\text { disperse swelling and dissipate binds }\end{array}$ \\
\hline & & Pharmaceutical study: (1) Purgative effect \\
\hline \multirow[t]{2}{*}{ Asari Radix et rhizama } & \multirow[t]{2}{*}{17.69} & $\begin{array}{l}\text { TCM: (1) To dispel wind and dissipate cold, (2) to dispel } \\
\text { wind and relieve pain, (3) To open the orifices, (4) To } \\
\text { warm the lung and resolve fluid retention }\end{array}$ \\
\hline & & $\begin{array}{l}\text { Pharmaceutical study: (1) Sedative and analgesic effects, } \\
\text { (2) Antipyretic and anti-inflammatory effects, (3) } \\
\text { Respiratory effect, (4) Cardiovascular effect, (5) } \\
\text { Anti-histaminic and anti-allergic effects }\end{array}$ \\
\hline \multirow[t]{2}{*}{$\begin{array}{l}\text { Synthesized Moschus } \\
\text { Artifactus }\end{array}$} & \multirow[t]{2}{*}{0.45} & $\begin{array}{l}\text { TCM: (1) To induce resuscitation and restore } \\
\text { consciousness, (2) To promote blood circulation to } \\
\text { remove obstruction, (3) To alleviate pain }\end{array}$ \\
\hline & & $\begin{array}{l}\text { Pharmaceutical study: (1) Effects on the CNS, (2) } \\
\text { cardiovascular effects, (3) Anti-inflammatory effect, (4) } \\
\text { Effects on the uterus }\end{array}$ \\
\hline \multirow[t]{2}{*}{$\begin{array}{l}\text { Zingiberis Rhizoma Recens } \\
\text { (fresh ginger juice) }\end{array}$} & \multirow[t]{2}{*}{50} & $\begin{array}{l}\text { TCM: (1) To release the exterior and dissipate cold, (2) } \\
\text { To warm the middle energizer to check vomiting, (3) To } \\
\text { resolve phlegm and suppress cough, (4) To reduce toxin } \\
\text { of fishery product }\end{array}$ \\
\hline & & $\begin{array}{l}\text { Pharmaceutical study: (1) Effects on the digestive } \\
\text { system, (2) Antiemetic effect, (3) Effect on the CNS }\end{array}$ \\
\hline
\end{tabular}

The first four herbs were grounded and mixed into a powder, with the ratio of $20 \%$ Bai Jie Zi, 25\% Yan Hu Suo, $15 \%$ Zhi Gan Sui, and 40\% Xi Xin. Then the fresh ginger juice was added into the mixed powder according to the ratio of $25 \mathrm{~mL}$ to $20 \mathrm{~g}$. Afterwards, the mixture was processed into $1 \mathrm{~cm}^{2}$ round patches weighing $2 \mathrm{~g}$ each. Finally, $0.02 \mathrm{~g}$ Artificial She Xiang was put on top of each patch. The placebo patch was made of flour and edible pigments. The substances will be mixed with water and made into similar $1 \mathrm{~cm}^{2}$ round patches weighing $2 \mathrm{~g}$ to achieve the similarity of the color and shape with Chinese Medicine patches.

Acupoints. Based on previous systematic review, preliminary survey among experienced TCM practitioners, and routine TJ regimen applied in HKBU clinics, Dazhui (GV 14), bilateral Feishu (UB 13), and bilateral Shenshu (UB 23), five acupoints in total were selected $[21,25,26]$. Participants in TJ group and placebo group received a 4 -week continuous treatment with either Chinese Medicine patches or placebo patches applied to each acupoint weekly, and then underwent a 4-week post-treatment follow-up. Patients in the waitlist group were not allowed getting any treatment during the same period but would be given compensatory TJ treatment in next 4 weeks. A $2 \mathrm{~cm} * 2 \mathrm{~cm}$ piece of hypoallergenic tape was utilized to paste the patches to each acupoint. The retention time was 2 hours. All operations were completed by our TCM practitioners who should be registered TCM practitioners with no less than 5 years of TCM undergraduate education and more than 5 years of clinical experience. The patching time could be properly shortened if the patient could not tolerate the stimulation or appeared allergic reactions. Names and details of acupoints were presented in Table 2.

2.4. Outcomes. The primary outcome was set to be the difference in the weekly average of the TNSS documented in patients' diaries aftertreatment. Four nasal symptoms, 
TABLE 2: Acupuncture points used in the study.

\begin{tabular}{|c|c|c|c|c|}
\hline Name & Areas & Special Qualification & Effects of Stimulation & Indications \\
\hline $\begin{array}{l}\text { Dazhui } \\
\text { (GV 14) }\end{array}$ & $\begin{array}{l}\text { In a depression below the } \\
\text { processus spinosus of the } \\
7^{\text {th }} \text { cervical and above the } \\
1^{\text {st }} \text { thoracic vertebrae. }\end{array}$ & $\begin{array}{l}\text { A copulo-conventory by } \\
\text { which the leading sinartery } \\
\text { communicates with all yang } \\
\text { conduits. }\end{array}$ & $\begin{array}{l}\text { Stabilising and regulating the } \\
\text { qi of centre; strengthening the } \\
\text { hepatic and pulmonal orb, } \\
\text { keeping the nasal open. }\end{array}$ & $\begin{array}{l}\text { Cough and } \\
\text { asthma }\end{array}$ \\
\hline $\begin{array}{l}\text { Feishu } \\
\text { (UB 13) }\end{array}$ & $\begin{array}{l}1.5 \text { PI laterally of the } \\
\text { processus spinosus of the } \\
3^{\text {rd }} \text { thoracic vertebrae }\end{array}$ & $\begin{array}{l}\text { Dorsal inductor } \\
\text { (inductorium dorsale) for } \\
\text { the pulmonal orb. }\end{array}$ & $\begin{array}{l}\text { Stabilising and harmonizing } \\
\text { the pulmonal and the renal } \\
\text { orb; regulating the qi; } \\
\text { strengthening the yin. }\end{array}$ & $\begin{array}{l}\text { Cough with } \\
\text { asthma, common } \\
\text { cold, nasal } \\
\text { congestion }\end{array}$ \\
\hline $\begin{array}{l}\text { Shenshu } \\
\text { (UB 23) }\end{array}$ & $\begin{array}{l}\text { 1.5 PI laterally of the } 2^{\text {nd }} \\
\text { lumbar vertebrae }\end{array}$ & $\begin{array}{l}\text { Dorsal inductor of the renal } \\
\text { orb. }\end{array}$ & $\begin{array}{l}\text { Strengthening and } \\
\text { harmonizing the renal orb; } \\
\text { improving hearing and } \\
\text { clearing vision. }\end{array}$ & $\begin{array}{l}\text { Cough, asthma, } \\
\text { asthenic } \\
\text { breathing }\end{array}$ \\
\hline
\end{tabular}

namely, rhinorrhea, nasal itching, nasal obstruction, and sneezing, compose the TNSS. A five-point scale from 0 to 4 $(0=$ no symptoms; $1=$ mild; $2=$ moderate $3=$ severe $; 4=$ very severe) was applied to evaluate the severity of the symptoms. The TNSS was the summation of four individual symptom scores, ranging from 0 (no symptom) to 16 (maximum symptom intensity). This scoring system has been generally utilized in efficacy evaluation of AR treatment [27, 28].

The secondary outcomes included the assessment of QoL and the rescue medication needs. The QoL was evaluated by using RQLQ, which has 28 questions in seven domains (sleep, non-rhinoconjunctivitis symptoms, practical problems, nasal symptoms, ocular symptoms, activity limitations, and emotional function) ranging from 0 (no impairment) to 6 (maximum impairment) [29]. The rescue medication needs were assessed by using rescue medication score (RMS). RMS was calculated daily on a 4 -point scale $(0=$ no rhinitis medicine; 1 = cetirizine, $10 \mathrm{mg} / \mathrm{d}$, or equivalent; 2 = cetirizine, $20 \mathrm{mg} / \mathrm{d}$, or equivalent; 3 = systemic or topical corticosteroids for nose or lung) (daily range; 0 to 3; weekly range: 0 to 21 ). When more than one rescue medication was applied in one day, only the highest level medication was documented [30].

Adverse events were collected throughout the study, on the foundation of participant-reports and practitioners' consultation. If serious adverse events occurred, the treatment would be suspended immediately, and whether the trial should be suspended would be decided after further assessment.

2.5. Randomization Assignment. This study was a randomized controlled trial. Qualified participants were randomly distributed to TJ arm, placebo arm or waitlist arm according to the ratio of 1:1:1. Random Allocation Software (version 1.0.0, Isfahan, Iran) was utilized to generate a randomization scheme. The principal investigator (PI) generated the random allocation sequence and was not allowed to inform other researchers. After the run-in stage, a research assistant (RA) assigned the treatments based on the codes, which were preserved in opaque sealed envelopes with consecutive randomization numbers. Emergency unblinding only happened when the intervention details were necessary for the patient's future management. Such arrangement would ensure the clinical evaluator and subjects to be blind to the allocation.

2.6. Sample Size Calculation. Sample size was estimated according to the primary outcome. As this was a pilot clinical trial, we assumed the improvement rate of waitlist group would be 0 . Afterwards, we supposed that there would be $30 \%$ improvement of TNSS in TJ group and $15 \%$ in placebo group. Considering a power of $80 \%$, and $\alpha$ value of $2.5 \%$ (twotailed), the sample size will be calculated using the following formula:

$$
n=\frac{2 \lambda}{\left(2 \sin ^{-1} \sqrt{P t}-2 \sin ^{-1} \sqrt{P w}\right)^{2}}
$$

The $\lambda$ was the cut-off value. $\mathrm{Pt}$ is $30 \%$ and $\mathrm{Pw}$ is 0 off value. 38 subjects in each were needed for the detection of significance [31]. Considering of a $20 \%$ dropout, the sample size was increased to 138 in total, with 46 participants in each arm.

2.7. Data Processing and Analysis. All efficacy and safety analyses were conducted based on intention-to-treat (ITT) principle. Missing values were imputed by the last-observationcarried-forward method. The statistical analysis was performed using the Statistical Packages of Social Sciences (SPSS) for Windows version 21.0. The statistical significance was defined as two-sided P-value of $<0.05$. Baseline characteristics were reported as mean (standard deviation (SD)) or frequency. Baseline differences among the groups were evaluated with the application of analysis of variance (ANOVA) for normally distributed continuous variables and non-parametric Kruskal-Wallis $H$ test for nonnormally distributed variables. For categorical variables, chisquared test or Fisher's exact test was applied. Comparisons between groups were conducted by using an analysis of covariance (ANCOVA) with baseline as covariate. All items and subscales were compared between groups for each 4-week using ANCOVA, with treatment group as a factor in the model and baseline as the covariate. The differences from baseline to the end of treatment in scores were tested with repeated measure ANOVA. 
TABLE 3: Baseline characteristics.

\begin{tabular}{|c|c|c|c|c|}
\hline Characteristic & $\begin{array}{l}\text { Tian Jiu } \\
(\mathrm{N}=46)\end{array}$ & $\begin{array}{c}\text { Sham } \\
\text { Tian Jiu } \\
(\mathrm{N}=46)\end{array}$ & $\begin{array}{l}\text { Waitlist } \\
\text { Tian Jiu } \\
(\mathrm{N}=44)\end{array}$ & $\mathrm{P}$ Value \\
\hline \multicolumn{5}{|l|}{ Sex, $n(\%)$} \\
\hline Female & $23(50.0)$ & $25(54.3)$ & $20(47.8)$ & \multirow{2}{*}{0.502} \\
\hline Male & $23(50.0)$ & $21(45.7)$ & $24(52.2)$ & \\
\hline Age, Mean (SD), y & $45.6(15.1)$ & $40.4(15.6)$ & $38.6(14.4)$ & 0.807 \\
\hline Weight, Mean (SD), kg & $64.9(19.7)$ & $64.8(21.3)$ & $59.8(15.0)$ & 0.878 \\
\hline Height, Mean (SD), cm & $165.2(11.3)$ & $161.6(23.6)$ & $160.1(15.9)$ & 0.400 \\
\hline BMI, Mean (SD), kg/m2 & $22.5(3.2)$ & $23.1(3.0)$ & $22.2(3.9)$ & 0.704 \\
\hline Duration of AR, $y$ & $17.1(11.8)$ & $15.0(8.1)$ & $14.6(10.1)$ & 0.280 \\
\hline TNSS, Mean (SD) & $10.37(3.4)$ & $9.25(3.05)$ & $9.38(2.57)$ & 0.664 \\
\hline Rhinorrhea & $2.52(1.04)$ & $2.36(1.08)$ & $2.03(1.07)$ & 0.118 \\
\hline Nasal itching & $2.78(1.06)$ & $2.52(1.17)$ & $2.6(1.06)$ & 0.357 \\
\hline Nasal obstruction & $2.33(1.22)$ & $2.18(1.08)$ & $2.48(1.09)$ & 0.803 \\
\hline Sneezing & $2.74(1.17)$ & $2.18(1.06)$ & $2.28(0.99)$ & 0.504 \\
\hline RQLQ, Mean(SD) & $2.32(1.35)$ & $1.91(1.48)$ & $1.49(1.13)$ & 0.349 \\
\hline Sleep & $2.48(1.46)$ & $2.23(1.81)$ & $1.92(1.59)$ & 0.491 \\
\hline Non-rhinoconjunctivitis symptoms & $2.44(1.27)$ & $2.05(1.55)$ & $1.65(1.11)$ & 0.103 \\
\hline Practical problems & $2.83(1.27)$ & $2.48(1.49)$ & $1.96(1.26)$ & 0.413 \\
\hline Nasal symptoms & $2.77(1.32)$ & $2.41(1.33)$ & $1.81(0.97)$ & 0.317 \\
\hline Ocular symptoms & $1.89(1.59)$ & $1.36(1.31)$ & $0.99(1.07)$ & 0.771 \\
\hline Activity limitations & $2.06(1.13)$ & $1.62(1.29)$ & $1.19(0.90)$ & 0.096 \\
\hline Emotional function & $1.82(1.38)$ & $1.23(1.60)$ & $0.91(1.00)$ & 0.646 \\
\hline
\end{tabular}

Within group differences were evaluated with ANOVA and post hoc comparison for normally distributed data and Wilcoxon signed-rank test for non-normally distributed data.

2.8. Data Collection and Handling of Withdrawal and Dropout. This was a 9-week clinical study, in which participants required to receive TJ treatment for 4 weeks, attend five assessment visits, finish a series of questionnaires, and avoid using other symptomatic treatments. All collected information was input into electronic file by a RA. Simultaneously, all files were stored in numerical order and kept in storage for 5 years. In order to ensure the maximum compliance, before the treatment, a consent process was conducted to inform the study arrangements, potential adverse events, and participants' responsibilities to all patients. Additionally, a RA would contact the participant to re-confirm the schedule before every visit. When a participant showed signs of withdrawl, we would try to explore the potential causes and seek the suitable solutions to ensure the compliance.

\section{Results}

3.1. Baseline Characteristics. From May 2016 to October 2016, 245 participants were screening and 138 participants were randomized. The flow diagram of patients was showed in
Figure 1. The baseline characteristics of three groups were presented in Table 3. The distributions of the demographic and clinical characteristics were well balanced and homogeneous $(P>0.05)$.

3.2. TNSS Change. After 4-week treatment, the total TNSS in TJ group was significantly decreased from 10.37 (SD: 3.40) to 6.24 (SD: 2.94). The change had statistical difference compared with waitlist group $(\mathrm{P}=0.003)$, while showing no statistical difference compared with placebo group (9.25 (SD: 3.05 ) to 6.63 (SD: 3.49), $\mathrm{P}=0.466$ ) (Figure 2). Among the four elements, the change of nasal obstruction score had statistical difference compared with the placebo group after 4-week treatment (from 2.33 (SD: 1.22) to 1.57 (SD: 0.83) compared with from 2.18 (SD: 1.08 ) to 1.78 (SD: 1.17), $\mathrm{P}=0.046$ ), and the effect on nasal obstruction remained in the 4-week followup (from 2.33 (SD: 1.22) to 1.43 (SD: 0.96) compared with from 2.18 (SD: 1.08) to 1.48 (SD: 1.05), $\mathrm{P}=0.044$ ) (Figure 2). Additionally, the change of nasal itching score showed no statistical difference compared with the placebo group (from 2.78 (SD: 1.06) to 1.74 (SD: 1.20) compared with from 2.52 (SD: 1.17 ) to 1.71 ( $\mathrm{SD}: 1.15), \mathrm{P}=0.073)$; however, the effect revealed in the 4-week follow-up (from 2.78 (SD: 1.06) to 1.52 (SD: $0.90)$ compared with from 2.52 (SD: 1.17) to 1.68 (SD: 1.09), $\mathrm{P}=0.021$ ) (Figure 2). Besides, the score of each symptom in TJ group was significantly reduced compared with waitlist group (Figure 2). The detailed changes of TNSS were shown in Table 4. 


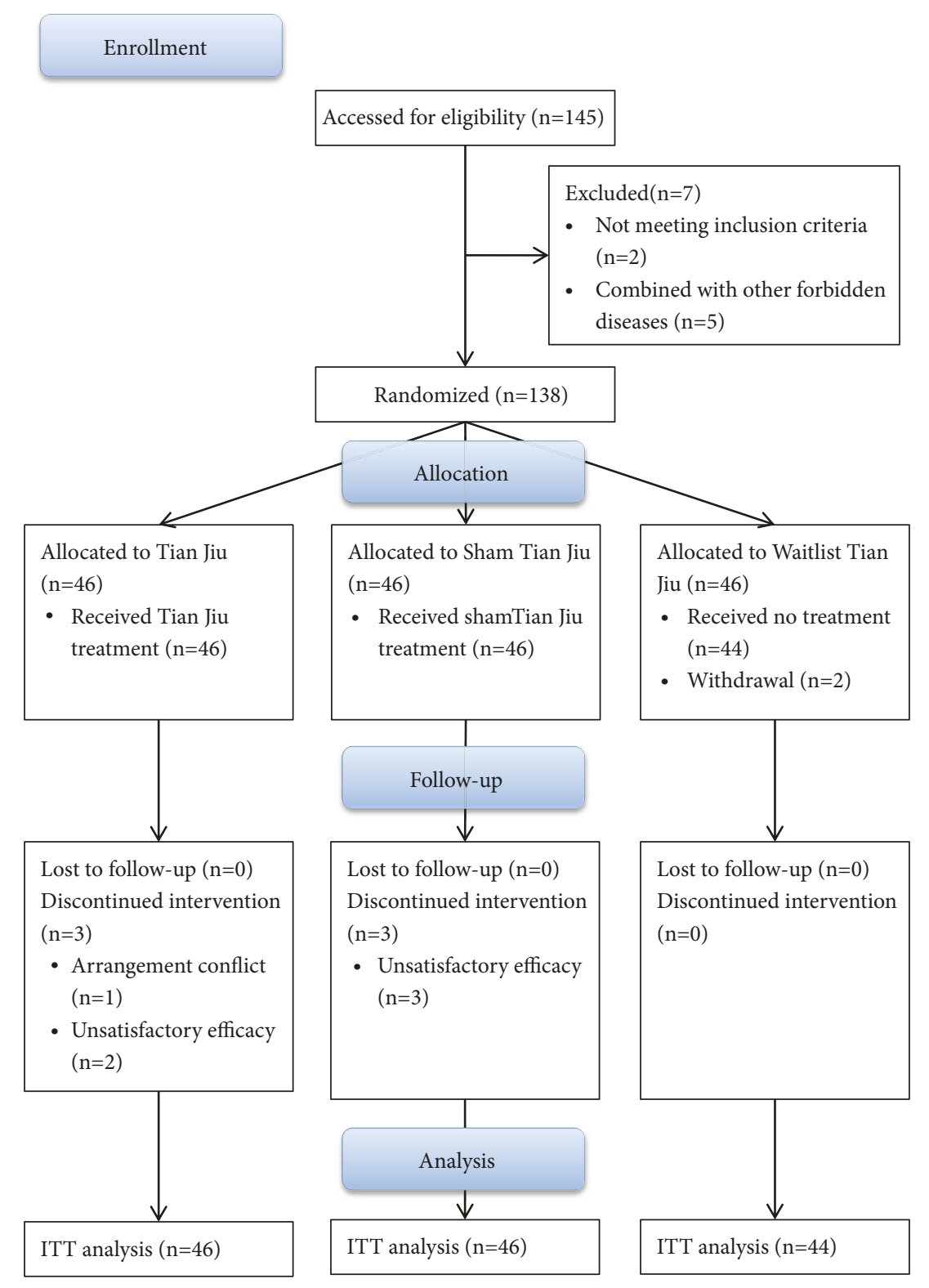

Figure 1: Participants flow diagram.

3.3. RQLQ Change. After 4-week treatment, the total RQLQ score in TJ group reduced from 2.32 (SD: 1.35) to 1.82 (SD: 1.19), while the placebo group and waitlist group only showed the trend of fluctuation (1.91 (SD: 1.48) to 1.95 (SD: 1.49) in placebo group and 1.49 (SD: 1.13) to 1.49 (SD: 1.13) in waitlist group). The difference compared with either group had statistical significance $(\mathrm{P}=0.006$ and $\mathrm{P}=0.008$, respectively). Moreover, the TJ group remained the slight decreasing trend in the 4-week follow-up (from 1.82 (SD: 1.19) to 1.80 (SD: 1.19), and the change had statistical significance compared with placebo group $(\mathrm{P}=0.009)$. With regard to the seven domains in RQLQ, after 4-week treatment, the TJ therapy benefited non-rhinoconjunctivitis symptoms, practical problems, nasal symptoms and activity limitations compared with nonintervention (waitlist group), and it showed superior efficacy than the placebo in sleep, non-rhinoconjunctivitis symptoms, nasal symptoms, and activity limitations. Then after 4week follow-up, the TJ therapy still showed advantages in sleep, non-rhinoconjunctivitis symptoms, practical problems and nasal symptoms compared with placebo. The detailed changes of RQLQ were shown in Table 5.

3.4. Safety Assessments. There were no serious adverse events that happened during the whole treatment. The common adverse events included flush, pruritus, blister, and pigmentation, occurring in 17, 23, 3, and 36 person-times among TJ group, and 3, 7, 1, and 4 person-times among placebo group, respectively. These adverse events were generally tolerated and disappeared quickly after removing the patches. For the rescue medication needs, the RMS could not be calculated 

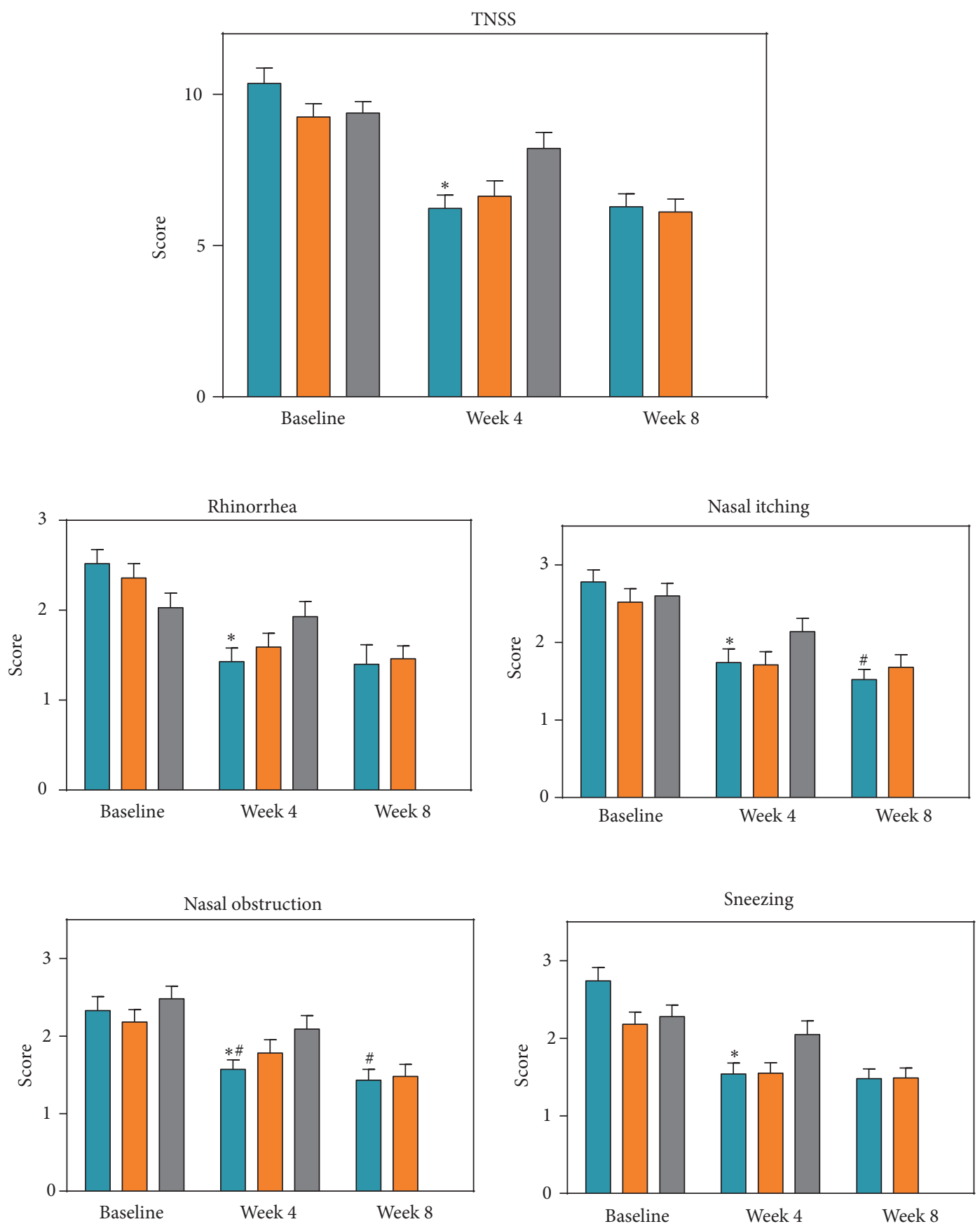

Tian Jiu

Sham Tian Jiu

$\square$ Waitlist Tian Jiu

$*$ Difference versus waitlist group, $\mathrm{P}<0.05$;

\#Difference versus waitlist group, $\mathrm{P}<0.05$.

FIGURE 2: Comparison of TNSS between three groups. 
TABLE 4: The TNSS in different visits.

\begin{tabular}{|c|c|c|c|c|c|}
\hline & $\begin{array}{l}\text { Tian Jiu } \\
(\mathrm{N}=46)\end{array}$ & $\begin{array}{c}\text { Sham } \\
\text { Tian Jiu } \\
(\mathrm{N}=46)\end{array}$ & $\begin{array}{c}\text { Tian Jiu vs } \\
\text { Sham Tian } \\
\text { Jiu } \\
\text { P-value* }\end{array}$ & $\begin{array}{l}\text { Waitlist } \\
\text { Tian Jiu } \\
(\mathrm{N}=44)\end{array}$ & $\begin{array}{c}\text { Tian Jiu vs } \\
\text { Waitlist } \\
\text { Tian Jiu } \\
\text { P-value* }\end{array}$ \\
\hline \multicolumn{6}{|l|}{ TNSS } \\
\hline Baseline & $10.37(3.40)$ & $9.25(3.05)$ & - & $9.38(2.57)$ & - \\
\hline Week 4 & $6.24(2.94)$ & $6.63(3.49)$ & 0.466 & $8.21(3.56)$ & 0.003 \\
\hline Week 8 & $6.28(2.95)$ & $6.11(2.95)$ & 0.582 & - & - \\
\hline \multicolumn{6}{|l|}{ Rhinorrhea } \\
\hline Baseline & $2.52(1.04)$ & $2.36(1.08)$ & - & $2.03(1.07)$ & - \\
\hline Week 4 & $1.43(1.03)$ & $1.59(1.05)$ & 0.162 & $1.93(1.11)$ & 0.017 \\
\hline Week 8 & $1.40(1.01)$ & $1.46(0.98)$ & 0.115 & - & - \\
\hline \multicolumn{6}{|l|}{ Nasal itching } \\
\hline Baseline & $2.78(1.06)$ & $2.52(1.17)$ & - & $2.6(1.06)$ & - \\
\hline Week 4 & $1.74(1.20)$ & $1.71(1.15)$ & 0.073 & $2.14(1.13)$ & 0.016 \\
\hline Week 8 & $1.52(0.90)$ & $1.68(1.09)$ & 0.021 & - & - \\
\hline \multicolumn{6}{|c|}{ Nasal obstruction } \\
\hline Baseline & $2.33(1.22)$ & $2.18(1.08)$ & - & $2.48(1.09)$ & - \\
\hline Week 4 & $1.57(0.83)$ & $1.78(1.17)$ & 0.046 & $2.09(1.16)$ & 0.011 \\
\hline Week 8 & $1.43(0.96)$ & $1.48(1.05)$ & 0.044 & - & - \\
\hline \multicolumn{6}{|l|}{ Sneezing } \\
\hline Baseline & $2.74(1.17)$ & $2.18(1.06)$ & - & $2.28(0.99)$ & - \\
\hline Week 4 & $1.54(0.96)$ & $1.55(0.91)$ & 0.804 & $2.05(1.16)$ & 0.001 \\
\hline Week 8 & $1.48(0.86)$ & $1.49(0.86)$ & 0.062 & - & - \\
\hline
\end{tabular}

For each variable, the values are expressed as the mean (SD).

* Comparison based on the change of score.

because only few participants took emergency medications. To be specific, during the 4 -week treatment, 2 person-times used medication equivalent to cetirizine $10 \mathrm{mg} / \mathrm{d}$, and 1 person-time used systemic or topical corticosteroids for nose or lung in TJ group. The frequency and categories of applied rescue medications were exactly the same in placebo group. Then during the 4-week follow-up, the frequencies of using medication equivalent to cetirizine $10 \mathrm{mg} / \mathrm{d}$ were 3 and 2 in TJ group and placebo group, respectively, which also showed no distinct difference.

\section{Discussion}

TJ therapy is a typical external intervention of TCM and has been still widely utilized in clinical practice in China. Our results demonstrated certain effect of TJ therapy in improving nasal obstruction and quality of life of AR patients during treatment period, despite the total TNSS of TJ group did not show significant difference compared with placebo. In addition, the treatment effect could remain after the TJ therapy withdrawal. The study presented relatively consistent results of previous studies $[21,25,26]$.

TJ therapy has a long history in clinical practice. The ancient classic "Zhang Shi Yi Tong” in Qing Dynasty firstly documented using Bai Jie Zi patching to treat cold wheezing. According previous statistics, AR ranked the third among the most commonly diseases treated by TJ therapy [11]. Comparing with other TCM external interventions, TJ therapy has its own advantages. For example, TJ therapy is easier to be self-handled. Unlike acupuncture and moxibustion, TJ could be completed by patients themselves if detailed patching locations are given. In addition, the adverse events are slight. As shown in our results, the major adverse events would disappear quickly without any special managements. Relatively, the risk of adverse events of other interventions such as acupuncture was higher, including bleeding and stabbing pain [32].

The basic treatment principles against $\mathrm{AR}$ include immune-regulation and anti-inflammation. Previous studies have also illustrated TJ's effects on these two aspects. The enhanced IgE level is a vital factor in AR pathogenesis. It could trigger the release of inflammatory mediators, such as histamine and leukotrienes, which would induce arteriolar dilation, vascular permeability elevation, itching, rhinorrhea (runny nose), mucous secretion, and smooth muscle contraction [33, 34]. Previous researches have found that TJ treatment could significantly decrease the serum IgE [35-38]. In addition, studies have also showed than TJ therapy could regulate the level of certain cytokines, for instance, decreasing interleukin-4 (IL-4), interleukin-5 (IL-5), and tumor necrosis factor- $\alpha$ (TNF- $\alpha)$ that may reduce the production of IgE from upstream [38]. Moreover, it has also been proven that 
TABLE 5: The RQLQ in different visits.

\begin{tabular}{|c|c|c|c|c|c|}
\hline & $\begin{array}{l}\text { Tian Jiu } \\
(\mathrm{N}=46)\end{array}$ & $\begin{array}{l}\text { Sham } \\
\text { Tian Jiu } \\
(\mathrm{N}=46)\end{array}$ & $\begin{array}{c}\text { Tian Jiu vs } \\
\text { Sham Tian } \\
\text { Jiu } \\
\text { P-value* }\end{array}$ & $\begin{array}{l}\text { Waitlist } \\
\text { Tian Jiu } \\
(\mathrm{N}=44)\end{array}$ & $\begin{array}{c}\text { Tian Jiu vs } \\
\text { Waitlist } \\
\text { Tian Jiu } \\
\text { P-value* }\end{array}$ \\
\hline \multicolumn{6}{|l|}{ RQLQ } \\
\hline Baseline & $2.32(1.35)$ & $1.91(1.48)$ & & $1.49(1.13)$ & \\
\hline Week 4 & $1.82(1.19)$ & $1.95(1.49)$ & 0.006 & $1.49(1.13)$ & 0.008 \\
\hline Week 8 & $1.80(1.19)$ & $1.92(1.48)$ & 0.009 & - & - \\
\hline \multicolumn{6}{|l|}{ Sleep } \\
\hline Baseline & $2.48(1.46)$ & $2.23(1.81)$ & & $1.92(1.59)$ & \\
\hline Week 4 & $1.76(1.21)$ & $2.25(1.79)$ & 0.038 & $1.91(1.59)$ & 0.057 \\
\hline Week 8 & $1.77(1.21)$ & $2.19(1.79)$ & 0.027 & - & - \\
\hline \multicolumn{6}{|c|}{ Non-rhinoconjunctivitis symptoms } \\
\hline Baseline & $2.44(1.27)$ & $2.05(1.55)$ & & $1.65(1.11)$ & \\
\hline Week 4 & $1.90(1.24)$ & $2.11(1.57)$ & 0.016 & $1.64(1.11)$ & 0.014 \\
\hline Week 8 & $1.88(1.22)$ & $2.07(1.55)$ & 0.034 & - & - \\
\hline \multicolumn{6}{|c|}{ Practical problems } \\
\hline Baseline & $2.83(1.27)$ & $2.48(1.49)$ & & $1.96(1.26)$ & \\
\hline Week 4 & $2.40(1.32)$ & $2.56(1.46)$ & 0.609 & $1.99(1.26)$ & 0.018 \\
\hline Week 8 & $2.37(1.33)$ & $2.53(1.48)$ & 0.046 & - & - \\
\hline \multicolumn{6}{|c|}{ Nasal symptoms } \\
\hline Baseline & $2.77(1.32)$ & $2.41(1.33)$ & & $1.81(0.97)$ & \\
\hline Week 4 & $2.16(1.12)$ & $2.51(1.32)$ & 0.014 & $1.83(0.97)$ & 0.018 \\
\hline Week 8 & $2.14(1.12)$ & $2.49(1.34)$ & 0.046 & - & - \\
\hline \multicolumn{6}{|c|}{ Ocular symptoms } \\
\hline Baseline & $1.89(1.59)$ & $1.36(1.31)$ & & $0.99(1.07)$ & \\
\hline Week 4 & $1.32(1.09)$ & $1.3(1.30)$ & 0.394 & $0.98(1.08)$ & 0.370 \\
\hline Week 8 & $1.32(1.08)$ & $1.3(1.30)$ & 0.252 & - & - \\
\hline \multicolumn{6}{|c|}{ Activity limitations } \\
\hline Baseline & $2.06(1.13)$ & $1.62(1.29)$ & & $1.19(0.90)$ & \\
\hline Week 4 & $1.66(1.04)$ & $1.66(1.36)$ & 0.031 & $1.18(0.89)$ & 0.030 \\
\hline Week 8 & $1.64(1.02)$ & $1.59(1.29)$ & 0.052 & - & - \\
\hline \multicolumn{6}{|c|}{ Emotional function } \\
\hline Baseline & $1.82(1.38)$ & $1.23(1.60)$ & & $0.91(1.00)$ & \\
\hline Week 4 & $1.55(1.33)$ & $1.29(1.61)$ & 0.512 & $0.89(1.04)$ & 0.444 \\
\hline Week 8 & $1.47(1.32)$ & $1.25(1.62)$ & 0.140 & - & - \\
\hline
\end{tabular}

For each variable, the values are expressed as the mean (SD).

$*$ Comparison based on the change of score.

TJ treatment could downregulate the level of eosinophil cationic protein (ECP), which meant TJ also had certain anti-inflammation effect $[35,37]$. Our study did not involve any relevant blood tests to evaluate the immune state and inflammation level of participants. This should be filled up in future studies.

The treatment effect of TJ therapy could be affected by multiple factors, including treatment timing, frequency, retention time, and body response [39]. Our study conducted the intervention under the most basic conditions, namely, normal days, routine treatment sessions (4 times) and patching time ( 2 hours), and no strict body response requirements, therefore to evaluate the fundamental efficacy of TJ therapy. Further research is worth being conducted. Firstly, the treatment timing could be moved to Sanfu days (the hottest days of the year). Recent study has reported that TJ therapy in Sanfu days had better long-term efficacy than normal TJ therapy, which indicated that the therapeutic effect of Sanfu TJ therapy may remain longer [36]. Secondly, increasing treatment sessions and enhancing patching time could be considered. Xu et al. has found that there was a positive correlation between the efficacy of TJ therapy and treatment years. The longer the patients applied TJ therapy, the more stable the efficacy obtained [40]. In addition, 
referring to the published systematic review, most studies set the patching time as 4-6 hours, while our study only set 2 hours [21]. The extended retention time increased the duration of local irritations and then may contribute the efficacy. Besides, there has also been reported that blistering TJ had better efficacy than non-blistering TJ [41]. Nevertheless, considering that blistering $\mathrm{TJ}$ is a traumatic external intervention, the application may not be smooth.

Our study has several limitations. Although the pasting procedure of TJ patches was completed by our practitioners, the peeling was finished by participants themselves. Some participants may not tolerate the stimulation, or remember the time incorrectly. The patching time could be shortened or extended, then led to potential bias. Besides, the followup period was only 4 weeks, which was not enough to assess the long-term effect. The future study should consider at least 6-month follow-up period or even longer to evaluate the persistent effect. Thirdly, the sample size of this pilot study was calculated based on primary outcome. We assumed the waitlist group would have no improvement. However, after the analysis, surprisingly we found the TNSS in waitlist group had $12.4 \%$ decline. This effect may have resulted from the environment and/or psychological impact. This ignored effect did not involve in the sample size calculation and then may lead to the underestimation of needed participants.

\section{Conclusion}

In summary, this randomized, single-blinded, controlled trial served primary evidence of the efficacy and safety of TJ therapy on AR in Hong Kong. This pilot study provided a fundamental TJ protocol for future research. Through adjusting treatment timing, frequency, retention time, and even body response settings, it has the potential to develop into an optimal therapeutic method for future application.

\section{Data Availability}

The data used to support the findings of this study are available from the corresponding author upon request.

\section{Disclosure}

The funding body had no role in the protocol design, study implementation, data collection, and manuscript preparation. The funding body did not participat in any segments of this study.

\section{Conflicts of Interest}

The authors have no conflicts of interest in regard to this paper.

\section{Authors' Contributions}

Liang Dai and Linda LD Zhong drafted the manuscript and contributed equally. Liang Dai, Wai Kun, and Linda LD Zhong conducted the study. Wai Ching Lam and Zhen
Yang screened and assessed the participants. Huaixue $\mathrm{Mu}$ did the quality control analysis. Tao Huang and Linda LD Zhong analyzed the data. Zhao-xiang Bian designed the study and supervised the whole study. All the authors read and approved the manuscript.

\section{Acknowledgments}

The research was financially supported by the Marcoda Co., Ltd.

\section{Supplementary Materials}

Supplement 1. CONSORT 2010 Checklist. Supplement 2. STRICTOM checklist. Supplement 3. Calibration curves of references compounds determined. Supplement 4 . HPLC chromatograms. Supplement 5. The name, source, and contents of references compounds of each ingredient (Supplementary Materials)

\section{References}

[1] H. A. Kakli and T. D. Riley, "Allergic rhinitis," Primary Care: Clinics in Office Practice, vol. 43, no. 3, pp. 465-475, 2016.

[2] L. Zhang, D. Han, D. Huang et al., "Prevalence of self-reported allergic rhinitis in eleven major cities in China," International Archives of Allergy and Immunology, vol. 149, no. 1, pp. 47-57, 2009.

[3] X. Wang, M. Zheng, H. Lou et al., "An increased prevalence of self-reported allergic rhinitis in major Chinese cities from 2005 to 2011," Allergy, vol. 71, no. 8, pp. 1170-1180, 2016.

[4] M. Schatz, "A survey of the burden of allergic rhinitis in the USA," Allergy, vol. 62, suppl 85, pp. 9-16, 2007.

[5] G. W. Canonica, J. Bousquet, J. Mullol, G. K. Scadding, and J. C. Virchow, "A survey of the burden of allergic rhinitis in Europe," Allergy, vol. 62, suppl 85, pp. 17-25, 2007.

[6] P. J. Bousquet, P. Demoly, P. Devillier, K. Mesbah, and J. Bousquet, "Impact of allergic rhinitis symptoms on quality of life in primary care," International Archives of Allergy and Immunology, vol. 160, no. 4, pp. 393-400, 2013.

[7] V. Jaruvongvanich, P. Mongkolpathumrat, H. Chantaphakul, and J. Klaewsongkram, "Extranasal symptoms of allergic rhinitis are difficult to treat and affect quality of life," Allergology International, vol. 65, no. 2, pp. 199-203, 2016.

[8] L. M. Wheatley and A. Togias, "Allergic rhinitis," The New England Journal of Medicine, vol. 372, no. 5, pp. 456-463, 2015.

[9] P. White, H. Smith, N. Baker, W. Davis, and A. Frew, "Symptom control in patients with hay fever in UK general practice: How well are we doing and is there a need for allergen immunotherapy?" Clinical \& Experimental Allergy, vol. 28, no. 3, pp. 266-270, 1998.

[10] X.-M. Li, "Complementary and alternative medicine in pediatric allergic disorders," Current Opinion in Allergy and Clinical Immunology, vol. 9, no. 2, pp. 161-167, 2009.

[11] F. Zhou, D. Yang, J.-Y. Lu et al., "Characteristics of clinical studies of summer acupoint herbal patching: a bibliometric analysis," BMC Complementary and Alternative Medicine, vol. 15, no. 1, p. 381, 2015.

[12] Y. Y. Kung, Y. C. Chen, S. J. Hwang, T. J. Chen, and F. P. Chen, "The prescriptions frequencies and patterns of Chinese herbal 
medicine for allergic rhinitis in Taiwan," Allergy, vol. 61, no. 11, pp. 1316-1318, 2006.

[13] L. Wilcox, "San Fu moxibustion and lung-related disorders," Chinese Medical Journal, vol. 8, pp. 14-18, 2009.

[14] L. Deng, "Chinese acupuncture and moxibustion," Traditional Medicine in Asia, p. 75, 2001.

[15] F. Jiang, "Relationship between allergic rhinitis and Chinese medical constitution," China Journal of Traditional Chinese Medicine and Pharmacy, vol. 23, no. 2, pp. 140-142, 2008.

[16] P. Wei, W. Kou, C. Zhang et al., "Disscussion on mechanism of acupoint therapy for allergic rhinitis," Liaoning Journal of Traditional Chinese Medicine, vol. 10, pp. 60-64, 2015.

[17] J. Chen, T. Yang, X. S. Lai et al., "Effect of acupoints external application of herbal drug on blood cell classification and serum specific IgE level of allergic rhinitis mice," Journal of Guangzhou University of Traditional Chinese Medicine, vol. 25, no. 4, pp. 304-306, 2008.

[18] J. Chen, X. S. Lai, C. Z. Tang, L. L. He, and B. X. Jin, "Experimental study of the influence of point application on celiac mastocyte degranulation in allergic rrhinitis mice," Shanghai Journal of Acupuncture and Moxibustion, vol. 26, no. 12, pp. 4245, 2007.

[19] J. Chen, Y. R. Yue, and X. S. Lai, "Effect of acupoint external application of herbal drugs on pathological changes of nasal mucosa of allergic rhinitis mice," Journal of Guangzhou University of Traditional Chinese Medicine, vol. 23, no. 6, pp. 518-521, 2006.

[20] J. Chen, X. S. Lai, and C. Z. Tang, "Influence of acupoints application of herbal medicine on specific proliferation of spleen lymphocytes in allergic rhinitis mice," Journal of Guangzhou University of Traditional Chinese Medicine, vol. 28, no. 3, pp. 243-245, 2011.

[21] F. Zhou, L. Yan, G. Yang, and J. Liu, “Acupoint herbal patching for allergic rhinitis: a systematic review and meta-analysis of randomised controlled trials," Clinical Otolaryngology, vol. 40, no. 6, pp. 551-568, 2015.

[22] W. Kun, L. L. Zhong, L. Dai et al., "Tian Jiu therapy for allergic rhinitis: study protocol for a randomized controlled trial," Trials, vol. 17, no. 1, p. 248, 2016.

[23] K. F. Schulz, D. G. Altman, and D. Moher, "CONSORT 2010 statement: updated guidelines for reporting parallel group randomized trials," Annals of Internal Medicine, vol. 152, no. 11, pp. 726-732, 2010.

[24] C. Cheng, S. Fu, Q. Zhou et al., "Extending the CONSORT Statement to moxibustion," Journal of Integrative Medicine, vol. 11, no. 1, pp. 54-63, 2013.

[25] HKBU eNews, "Chinese medicine research finds "Tianjiu" helpful in alleviating asthma," 2010, http://enews.hkbu.edu.hk/ view_article.php?id=11539.

[26] HKBU eNews, "HKBU clinical study finds Tian Jiu treatment helps relieve allergic rhinitis and bronchial asthma," 2014, http://hkbuenews.hkbu.edu.hk/?t=enews_details/689.

[27] J. I. Kim, M. S. Lee, S. Y. Jung et al., "Acupuncture for persistent allergic rhinitis: a multi-centre, randomised, controlled trial protocol," Trials, vol. 10, no. 1, p. 54, 2009.

[28] C. C. Xue, X. An, T. P. Cheung et al., "Acupuncture for persistent allergic rhinitis: a randomised, sham-controlled trial," The Medical Journal of Australia, vol. 187, no. 6, pp. 337-341, 2007.

[29] E. F. Juniper, G. H. Guyatt, L. E. Griffith, and P. J. Ferrie, "Interpretation of rhinoconjunctivitis quality of life questionnaire data," The Journal of Allergy and Clinical Immunology, vol. 98, no. 4, pp. 843-845, 1996.
[30] J. Kuehr, J. Brauburger, S. Zielen et al., "Efficacy of combination treatment with anti-IgE plus specific immunotherapy in polysensitized children and adolescents with seasonal allergic rhinitis," The Journal of Allergy and Clinical Immunology, vol. 109, no. 2, pp. 274-280, 2002.

[31] H. Yan, Medical Statistics, People's Medical Publishing House, Beijing, China, 2nd edition, 2010.

[32] B. Brinkhaus, M. Ortiz, C. M. Witt et al., "Acupuncture in patients with seasonal allergic rhinitis: a randomized trial," Annals of Internal Medicine, vol. 158, no. 4, pp. 225-234, 2013.

[33] P. Small, S. Frenkiel, A. Becker et al., "Rhinitis: a practical and comprehensive approach to assessment and therapy," The Journal of Otolaryngology, vol. 36, no. S1, pp. S5-S27, 2007.

[34] M. S. Dykewicz and D. L. Hamilos, "Rhinitis and sinusitis," The Journal of Allergy and Clinical Immunology, vol. 125, no. 2, Suppl 2, pp. S103-S115, 2010.

[35] W.-H. Hsu, T.-J. Ho, C.-Y. Huang et al., "Chinese medicine acupoint herbal patching for allergic rhinitis: a randomized controlled clinical trial," American Journal of Chinese Medicine, vol. 38, no. 4, pp. 661-673, 2010.

[36] M. S. Fang, Y. C. Dou, and S. M. Yao, "Clinical research of medicinal vesiculation for perennial allergic rhinitis," Chinese Acupuncture and Moxibustion, vol. 34, no. 9, pp. 857-860, 2014.

[37] Y. Chen, Z. Wang, and W. Cong, "A randomized controlled trial of application in canicular days for allergic rhinitis," Shanghai Journal of Acupuncture and Moxibustion, vol. 35, no. 6, pp. 700703, 2016.

[38] Z.-H. Ke and S.-H. Long, "Medicinal vesiculation combined with quick cupping at Shenque (CV 8) for allergic rhinitis with syndrome of yang deficiency: a randomized controlled trial," Chinese Acupuncture and Moxibustion, vol. 34, no. 9, pp. 853856, 2014.

[39] M. Qiu, Science of Acupuncture and Moxibustion, Shanghai Scientific and Technical Publishers, Shanghai, China, 1985.

[40] Z. Xu, J. Luo, Z. Gu, and W. Fu, "Relationship between the therapeutic effect of dog-day auto-moxibustion and course in the treatment of allergic rhinitis," Journal of Traditional Chinese Medicine, vol. 30, no. 7, pp. 2490-2492, 2015.

[41] Q. Chen, Observation on the Efficacy of Treating Allergic Rhinitis with the Method of Moxibustion and Non-Foaming in the Day, Shandong University of Traditional Chinese Medicine, 2015. 


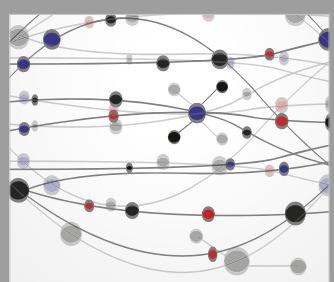

The Scientific World Journal
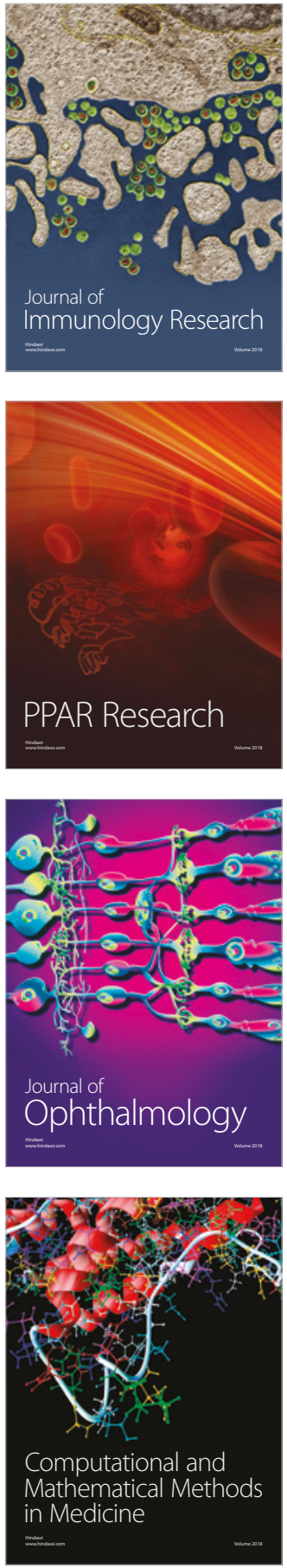

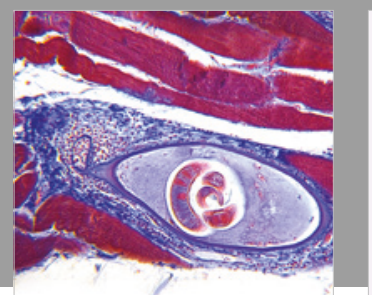

Gastroenterology Research and Practice

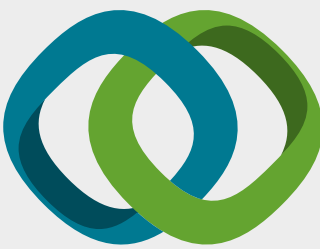

\section{Hindawi}

Submit your manuscripts at

www.hindawi.com
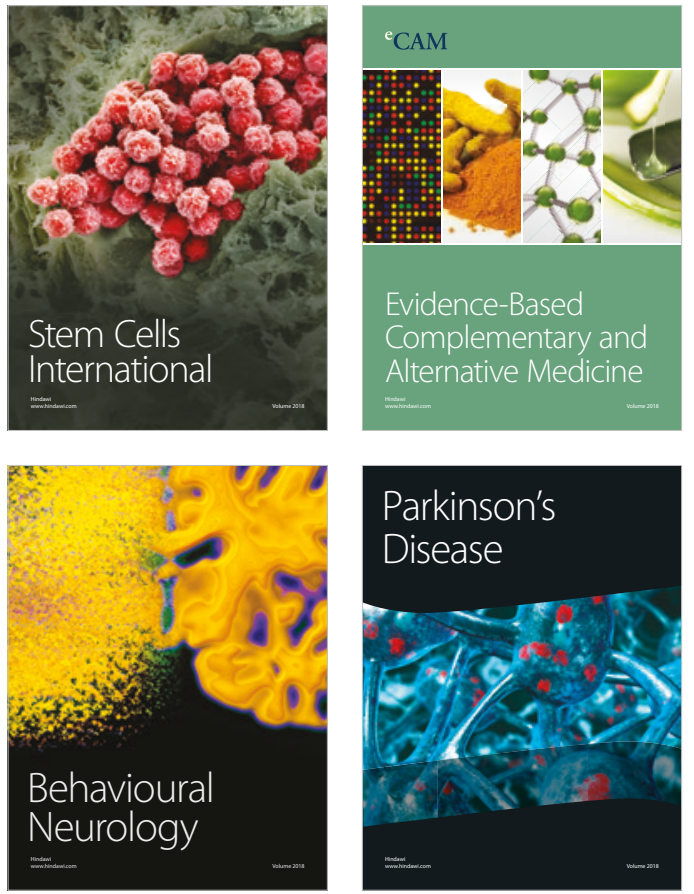

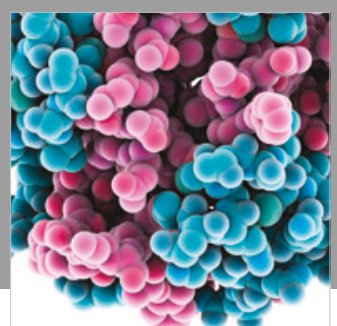

ournal of

Diabetes Research

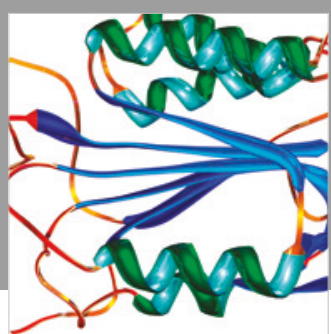

Disease Markers
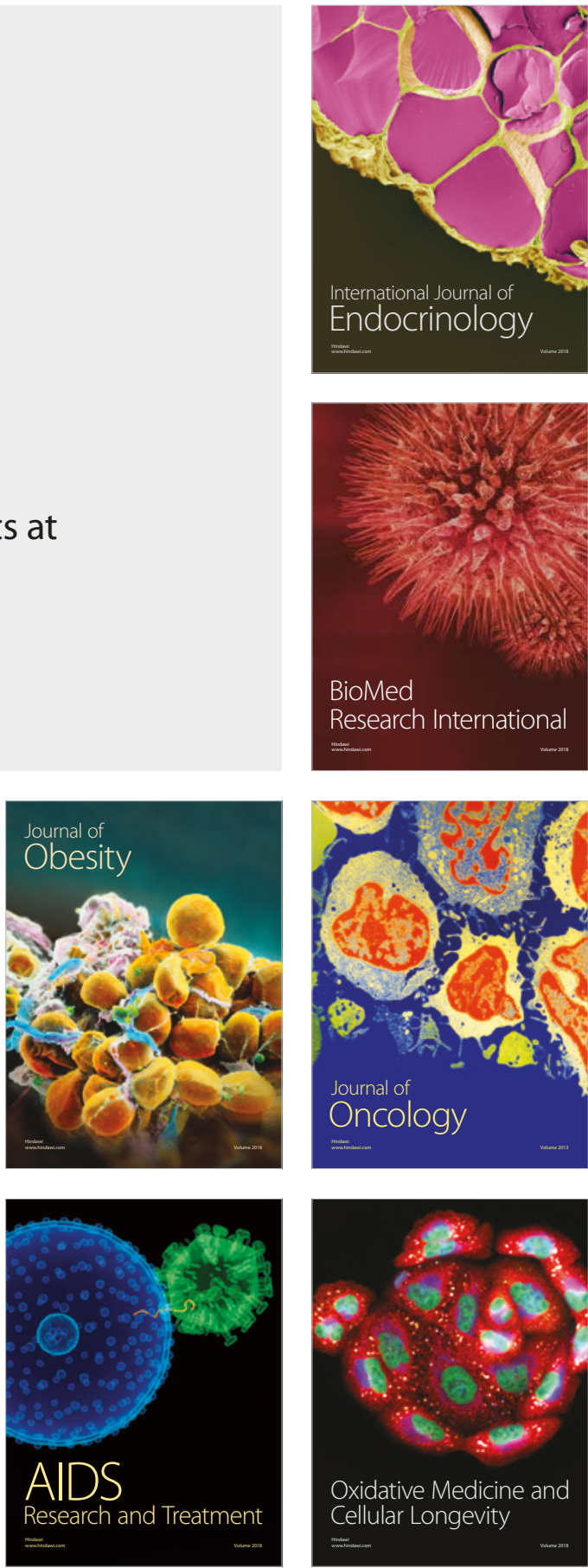